See discussions, stats, and author profiles for this publication at: https://www.researchgate.net/publication/342159539

\title{
A Tutorial on Joint Radar and Communication Transmission for Vehicular Networks -Part II: State of the Art and Challenges Ahead
}

Preprint · June 2020

\section{CITATIONS}

0

2 authors:

(2) Fan Liu

Southern University of Science and Technology

80 PUBLICATIONS 651 CITATIONS

SEE PROFILE

Some of the authors of this publication are also working on these related projects:

Wireless interference as a source of green signal power View project

[PAINLESS] Energy-autonomous portable access points for infrastructure-less networks View project
READS

126

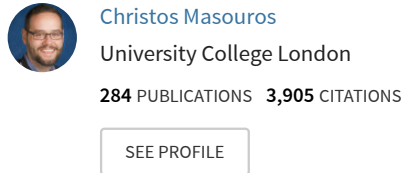




\title{
A Tutorial on Joint Radar and Communication Transmission for Vehicular Networks - Part II: State of the Art and Challenges Ahead
}

\author{
Fan Liu, Member, IEEE, and Christos Masouros, Senior Member, IEEE
}

(Invited Paper)

\begin{abstract}
In Part I of this three-part tutorial on dualfunctional radar-communication (DFRC), we overviewed the fundamental elements of DFRC. As Part II of the tutorial, this letter overviews the state-of-the-art (SoA) in DFRC, with a particular emphasis on the use of the technique for seamless connectivity in the vehicular network. We commence by introducing the conventional beam tracking approaches for millimeter wave (mmWave) communication systems, based exclusively on communication signalling and feedback, followed by the DFRC based schemes tailored for the vehicular network. Finally, we evaluate a number of SoA DFRC schemes through comparative simulations.
\end{abstract}

Index Terms-Dual-functional radar-communication, vehicular network, beam tracking, beam prediction

\section{INTRODUCTION}

$\mathbf{T}$ HE coming generations of vehicular networks, dominated by the connected and autonomous vehicle paradigm, will rely on seamless communication and radar intelligence. At present, however, communication and radar systems have to compete over a large portion of the wireless spectrum given the congestion in these frequency bands. In Part I of this tutorial, we presented the motivation behind DFRC as an enabler of the spectrum reuse between radar and communication transmission [1]. In vehicular networks, DFRC aims to jointly optimize vehicular communications with remote sensing capabilities for vehicle localization and anti-collision detection.

The next-generation vehicle-to-everything (V2X) network will require low-latency Gbps data transmission. While general communication technologies can cope with delays of hundreds of ms, V2X-controlled critical applications require delays of the order of tens of ms [2]. On the other hand, the vehicular network should also be equipped with the radar functionality to provide robust and high-resolution vehicle localization and environment sensing services [2] with signalling of extreme reliability. At the time of writing, vehicular localization and networking schemes are mostly realized by the Global Navigation Satellite System (GNSS) and default standards [3]. While these approaches do offer basic V2X functionalities, they suffer from poor sensing accuracy and low transmission data rate. To address the aforementioned issues, $5 \mathrm{G}$ technology utilizes both the massive multi-input multi-output (mMIMO) antenna array and the mmWave spectrum. By leveraging the advanced mMIMO beamforming techniques and the large bandwidth available at the mmWave spectrum, both the communication and the radar sensing capabilities can be greatly enhanced.
Given the inherent high-mobility features in the V2X network, it is essential to efficiently estimate the fast-changing vehicular channels, in order to guarantee the quality-of-service (QoS) for communication. In mmWave cellular systems, this is realized by sophisticated beam training and tracking techniques. Nevertheless, conventional beam training/tracking schemes are based on communication-only protocols, where frequent feedback is needed to configure the links, incurring considerable complexities, signalling overheads and latency. The motivation for integrating radar sensing and communication naturally arises in the vehicular network, which not only enhances the efficient utilization of both the spectral and signalling resources, but also enables the beneficial cooperation between the two functionalities. In particular, with the aid of the radar sensing, the pilots and the feedback loop required in the conventional mmWave beam tracking schemes [4], [5] are no longer needed, thus reducing the processing latency, which is key to vehicular network operated in the high-mobility environment.

In this letter, we first overview the conventional beam training and tracking approaches using communication-only protocols. We then overview the recent research framework on predictive beamforming conceived for the vehicle-toinfrastructure (V2I) network, which is built upon joint radarcommunication transmission. Moreover, we present a comparative evaluation between some indicative communication-only and DFRC techniques to reveal the usefulness of the latter in the V2X scenarios. Finally, we conclude Part II of this tutorial by identifying the open problems and challenges in this research area.

\section{BEAM TRAINING AND TRACKING}

\section{A. Beam Training}

The mmWave channel is known to be sparse in the angular domain, which translates to having only a few significant propagation paths. Therefore, to estimate the mmWave channel state information (CSI) is equivalent to estimating the angle of arrival (AoA), the angle of departure (AoD) and the channel gain of each path, which is typically termed as beam training. To this end, a straightforward idea is to fully scan the angular interval of interest with a collection of beams steered to different directions, and then choose those having the highest signalto-noise ratio (SNR) [6]. The SNR associated to different beams is measured at the receiver ( $\mathrm{Rx})$ side, and is fed back 


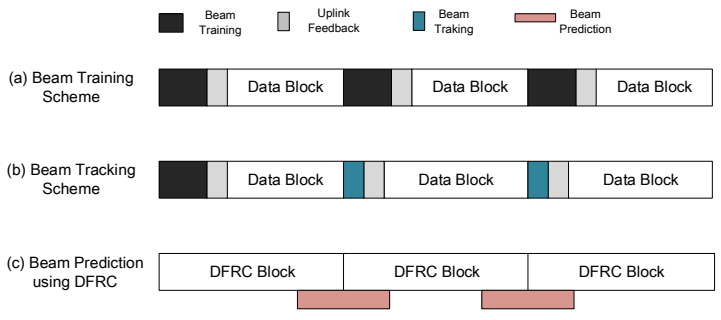

Fig. 1. Frame structure for mmWave beam training, tracking and prediction schemes.

to the transmitter (Tx) for processing. After obtaining the estimated angles, the Tx and the Rx will accordingly formulate transmit and receive beams, which are expected to be aligned with each other, such that a reliable communication link can be established. For clarity, we summarize the aforementioned procedure as a conceptual transmission protocol shown in Fig. 1(a). In each downlink block, the Tx first transmits pilots (beams) for beam training, and then transmits the data block by designing a transmit beamformer based on the beam information fed back from the Rx.

An important tradeoff that can be observed in Fig. 1(a) is the one between the estimation accuracy and the signalling overhead. Indeed, the optimal beam pairs can be more accurately acquired by transmitting more pilots, and thus improving the beamforming gain and SNR. However, this will inevitably occupy the temporal resources in data transmission, which incurs overhead and latency. As a result, the estimated CSI/trained beams could be easily outdated in high-mobility scenarios, which causes beam misalignment and degrades the performance. This is particularly pronounced for the mMIMO antenna array that formulates very narrow beams, where a small misalignment may cause notable performance loss. To tackle this issue, many efficient beam training schemes have been proposed in the literature, such as those relying on the adaptive compressed sensing (CS) algorithms [7], [8].

\section{B. Beam Tracking}

To further reduce the overhead in beam training, one may exploit the temporal correlation between the consecutive channel realizations. It has been shown that by using the previously estimated beams as the prior information, the number of pilots required can be minimized to an acceptable level. This technique, commonly referred to as beam tracking, has recently raised huge research interests in mobile mmWave communications [4], [9]. As shown in Fig. 1(b), the beam tracking technique requires an initial access/beam training at the beginning, and tracks the variation of the channel using only a few pilots in the subsequent transmission blocks. In the extreme case, only one or two pilots are needed in each block. In [5], the beam tracking is realized by extended Kalman filtering (EKF), where only a single downlink pilot is needed for each transmission. In [10], a fast beam tracking method has been proposed based on the auxiliary beam pair (ABP) design, where a pair of training beams are transmitted.

In what follows, we briefly discuss the ABP technique proposed in [10]. For the sake of convenience, let us consider a simple scenario where an $N_{t}$-antenna mMIMO base station (BS) is serving a single-antenna user in a line-of-sight (LoS) channel. Assume that the angle of the user at the $(n-1)$ th epoch is $\theta_{n-1}$, which is perfectly estimated and is known to the BS. Our aim is then to estimate $\theta_{n}$ based on $\theta_{n-1}$. By transmitting pilot symbols $x_{1}$ and $x_{2}$ using two beamformers $\mathbf{f}_{L}, \mathbf{f}_{R} \in \mathbb{C}^{N_{t} \times 1}$, the received signals at the user can be expressed as

$$
y_{1}=\alpha \mathbf{a}^{H}\left(\theta_{n}\right) \mathbf{f}_{L} x_{1}+z_{1}, y_{2}=\alpha \mathbf{a}^{H}\left(\theta_{n}\right) \mathbf{f}_{R} x_{2}+z_{2},
$$

where $\alpha$ is the channel coefficient of the $\operatorname{LoS}$ path, $\mathbf{a}(\theta) \in$ $\mathbb{C}^{N_{t} \times 1}$ is the steering vector of the $\mathrm{BS}$, which is assumed to be a uniform linear array (ULA), and finally $z_{1}$ and $z_{2}$ denote the Gaussian noise with the variance of $N_{0}$. By further assuming that the ULA has a half-wavelength inter-element spacing, a $(\theta)$ can be expressed as

$$
\mathbf{a}(\theta)=\sqrt{\frac{1}{N_{t}}}\left[1, e^{-j \pi \cos \theta}, \ldots, e^{-j \pi\left(N_{t}-1\right) \cos \theta}\right]^{T} .
$$

By introducing the notation $\vartheta=-\pi \cos (\theta)$, we have

$$
\mathbf{a}(\theta)=\mathbf{a}(\vartheta)=\sqrt{\frac{1}{N_{t}}}\left[1, e^{j \vartheta}, \ldots, e^{j\left(N_{t}-1\right) \vartheta}\right]^{T} .
$$

The beamformers $\mathbf{f}_{L}$ and $\mathbf{f}_{R}$ are designed based on the previously estimated beam direction $\vartheta_{n-1}$. The temporal correlation between $\vartheta_{n-1}$ and $\vartheta_{n}$ intuitively implies that, $\vartheta_{n}$ is not likely to be far away from $\vartheta_{n-1}$, and should be within a small interval centering on $\vartheta_{n-1}$, e.g., $\vartheta_{n} \in\left[\vartheta_{n-1}-\Delta \vartheta, \vartheta_{n-1}+\Delta \vartheta\right]$, with high probability. Therefore, the beamformers can be designed as

$$
\mathbf{f}_{L}=\mathbf{a}\left(\vartheta_{n}-\Delta \vartheta\right), \mathbf{f}_{R}=\mathbf{a}\left(\vartheta_{n}+\Delta \vartheta\right) .
$$

Without loss of generality, let the pilot symbols $x_{1}=x_{2}=1$. The user then estimates the SNR of $y_{1}$ and $y_{2}$, which are

$$
\gamma_{1}=\frac{|\alpha|^{2}\left|\mathbf{a}^{H}\left(\vartheta_{n}\right) \mathbf{f}_{L}\right|^{2}}{N_{0}}, \gamma_{2}=\frac{|\alpha|^{2}\left|\mathbf{a}^{H}\left(\vartheta_{n}\right) \mathbf{f}_{R}\right|^{2}}{N_{0}}
$$

By letting $\Delta \vartheta=\frac{2 k \pi}{N_{t}}, k \in\left\{1, \ldots, \frac{N_{t}}{4}\right\}$, the user calculates the following ratio metric [10]

$$
\lambda=\frac{\gamma_{1}-\gamma_{2}}{\gamma_{1}+\gamma_{2}}=-\frac{\sin \left(\vartheta_{n}-\vartheta_{n-1}\right) \sin (\Delta \vartheta)}{1-\cos \left(\vartheta_{n}-\vartheta_{n-1}\right) \cos (\Delta \vartheta)} .
$$

Since $\left|\vartheta_{n}-\vartheta_{n-1}\right| \leq \Delta \vartheta, \lambda$ is a monotonic function in $\vartheta_{n}-\vartheta_{n-1}$. One can readily solve for $\vartheta_{n}$ by using the following reverse function in the form

$\hat{\vartheta}_{n}=$

$\vartheta_{n-1}-\arcsin \left(\frac{\lambda \sin (\Delta \vartheta)-\lambda \sqrt{1-\lambda^{2}} \sin (\Delta \vartheta) \cos (\Delta \vartheta)}{\sin ^{2}(\Delta \vartheta)+\lambda^{2} \cos ^{2}(\Delta \vartheta)}\right)$.

Accordingly, $\theta_{n}$ can be obtained by $\hat{\theta}_{n}=\arccos \left(-\frac{\hat{\vartheta}_{n}}{\pi}\right)$. In the case that the SNR values $\gamma_{1}, \gamma_{2}$ are perfectly estimated, we have $\hat{\theta}_{n}=\theta_{n}$.

While the existing approaches can effectively track the beams by using a small number of pilots, frequent uplink feedbacks are still necessary. Moreover, the signal energy accumulated reduces when limiting the pilot overheads, which 


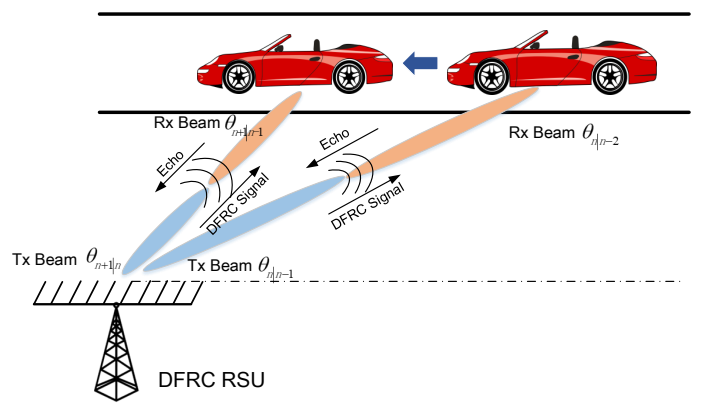

Fig. 2. DFRC transmission in the V2I scenario.

may potentially lead to estimation errors. More importantly, in fast-changing channels such as in the vehicular network, it is not sufficient to only track the beam. As a further step to adapt to the high mobility, the Tx should be able to predict the beam, such that the beamformers can be prepared in advance. In what follows, we overview a low-overhead predictive beamforming framework tailored for vehicular links based on the DFRC transmission [11], [12].

\section{Predictive Beamforming through DFRC: COMMUnication SERVED By RADAR SENSING}

Let us consider a mmWave road side unit (RSU) with $N_{t}$ transmit and $N_{r}$ receive antennas, which is serving an $N_{v^{-}}$ antenna vehicle driving at a nearly constant speed on a straight road, as shown in Fig. 2. For ease of notation, we assume that the RSU and the vehicle communicate via a LoS path, and that all the antenna arrays are ULAs and are adjusted to be parallel to the road. Consequently, the AoA equals to the AoD in the V2I LoS channel.

At each transmission block, the RSU formulates a narrow beam and transmits a DFRC signal $s(t) \in \mathbb{C}$ that contains data symbols intended for the vehicle at the time $t$. While being received by the vehicles's antenna, the signal is also reflected by the vehicle's body. At the $n$th epoch, denoting the vehicle's range, velocity and azimuth angle relative to the radar are $d_{n}$, $v_{n}$ and $\theta_{n}$, respectively, the received echo signal at the RSU can be expressed as

$\mathbf{r}_{n}(t)=\kappa \beta_{n} e^{j 2 \pi f_{D, n} t} \sqrt{p} \mathbf{b}\left(\theta_{n}\right) \mathbf{a}^{H}\left(\theta_{n}\right) \mathbf{f}_{n} s\left(t-\tau_{n}\right)+\mathbf{z}_{R, n}(t)$

where $\kappa=\sqrt{N_{t} N_{r}}$ denotes the array gain, $\beta_{n}$ contains both the radar cross-section (RCS) and the round-trip path-loss, $f_{D, n}=\frac{2 v_{n} \cos \theta_{n} f c}{c}$ is the Doppler frequency generated by the radial velocity, with $f_{c}$ and $c$ being the carrier frequency and the speed of the light, $p$ is the transmit power, $\tau_{n}=\frac{2 d_{n}}{c}$ is the round-trip delay, $f_{n}$ is the transmit beamformer, $\mathbf{a}(\theta) \in$ $\mathbb{C}^{N_{t} \times 1}$ and $\mathbf{b}(\theta) \in \mathbb{C}^{N_{r} \times 1}$ are transmit and receive steering vectors, which are expressed in the same form of (2). Finally, $\mathbf{z}_{R, n}(t) \in \mathbb{C}^{N_{r} \times 1}$ stands for the Gaussian noise. On the other hand, the received signal at the vehicle can be modeled as

$c_{n}(t)=\alpha_{n} \sqrt{N_{t} N_{v}} \sqrt{p} \mathbf{w}_{n}^{H} \mathbf{u}\left(\phi_{n}\right) \mathbf{a}^{H}\left(\theta_{n}\right) \mathbf{f}_{n} s(t)+z_{C, n}(t)$,

where $\alpha_{n}$ is the channel coefficient, $\mathbf{w}_{n} \in \mathbb{C}^{N_{v} \times 1}$ is a receive beamformer, $\mathbf{u}(\phi) \in \mathbb{C}^{N_{v} \times 1}$ is the array response vector at the vehicle, and $\phi_{n}$ is the AoA of the DFRC signal, which is assumed to be the same with the $\operatorname{AoD} \theta_{n}$ according to the assumption of the paralleled antenna arrays. Finally, $z_{C, n}(t)$ represents the noise.

By receiving the target echo $\mathbf{r}_{n}(t)$, the RSU attempts to obtain the estimates of the vehicle's state, i.e., $\mathbf{x}_{n}=$ $\left[\theta_{n}, d_{n}, v_{n}, \beta_{n}\right]^{T}$. Based on that, as well as on the motion model of the vehicle, the RSU predicts $\mathbf{x}_{n+1}$ and $\mathbf{x}_{n+2}$, which are exploited for transmit beamforming at the RSU and receive beamforming at the vehicle, respectively. To begin with, the RSU should firstly matched-filter $\mathbf{r}_{n}(t)$ with a time-delayed and Doppler-shifted counterpart of $s(t)$ to obtain the estimated $\hat{\tau}_{n}$ and $\hat{f}_{D, n}$, which are used to compensate for $\mathbf{r}_{n}(t)$. After compensation, the output of the matched filter is given by

$$
\tilde{\mathbf{r}}_{n}=\beta_{n} \sqrt{G p} \kappa \mathbf{b}\left(\theta_{n}\right) \mathbf{a}^{H}\left(\theta_{n}\right) \mathbf{f}_{n}+\mathbf{z}_{\theta, n},
$$

where $\mathbf{z}_{\theta, n}$ represents the output noise, and $G$ is the matched filtering gain, which typically equals to the energy of $s(t)$. Together with the delay and the Doppler frequency, the RSU obtains the following measurements as [11]

$$
\begin{aligned}
& \mathbf{y}_{n}=\mathbf{h}\left(\mathbf{x}_{n}\right)+\tilde{\mathbf{z}}_{n} \\
& =\left\{\begin{array}{l}
\tilde{\mathbf{r}}_{n}=\beta_{n} \sqrt{G p} \kappa \mathbf{b}\left(\theta_{n}\right) \mathbf{a}^{H}\left(\theta_{n}\right) \mathbf{f}_{n}+\mathbf{z}_{\theta, n} \\
\hat{\tau}_{n}=\frac{2 d_{n}}{c}+z_{\tau, n} \\
\hat{f}_{D, n}=\frac{2 v_{n} \cos \theta_{n} f_{c}}{c}+z_{f, n}
\end{array}\right.
\end{aligned}
$$

where $z_{\tau, n}$ and $z_{f, n}$ are the estimation noise of $\tau_{n}$ and $f_{D, n}$, respectively. The measurements $\mathbf{y}_{n}$ will be input into a welldesigned extended Kalman filter to obtain the state estimate $\hat{\mathbf{x}}_{n}$. With the estimate at hand, the RSU predicts the states $\hat{\mathbf{x}}_{n+1 \mid n}$ and $\hat{\mathbf{x}}_{n+2 \mid n}$ of the vehicle at the next two epoches, using a state evolution model as follows [11]

$$
\begin{aligned}
& \mathbf{x}_{n}=\mathbf{g}\left(\mathbf{x}_{n-1}\right)+\boldsymbol{\omega}_{n} \\
& =\left\{\begin{array}{l}
\theta_{n}=\theta_{n-1}+d_{n-1}^{-1} v_{n-1} \Delta T \sin \theta_{n-1}+\omega_{\theta, n}, \\
d_{n}=d_{n-1}-v_{n-1} \Delta T \cos \theta_{n-1}+\omega_{d . n}, \\
v_{n}=v_{n-1}+\omega_{v, n}, \\
\beta_{n}=\beta_{n-1}\left(1+d_{n-1}^{-1} v_{n-1} \Delta T \cos \theta_{n-1}\right)+\omega_{\beta, n},
\end{array}\right.
\end{aligned}
$$

where $\Delta T$ is the length of each time slot, and $\omega_{n}$ denotes the state noise, which is generated by approximation and other systematic errors.

At the $(n+1)$ th epoch, the RSU formulates a beamformer based on $\hat{\theta}_{n+1 \mid n}$, which is

$$
\mathbf{f}_{n+1}=\mathbf{a}\left(\hat{\theta}_{n+1 \mid n}\right) .
$$

By applying $\mathbf{f}_{n+1}$, the RSU sends the DFRC signal $s_{n+1}(t)$ to the vehicle, which contains both the data information and the predicted angle information $\hat{\theta}_{n+2 \mid n}$. At the $(n+2)$ th epoch, the vehicle formulates the receive beamformer based on $\hat{\theta}_{n+2 \mid n}$, which is

$$
\mathbf{w}_{n+2}=\mathbf{u}\left(\hat{\theta}_{n+2 \mid n}\right) .
$$

By iteratively estimating and predicting the vehicle's parameters at the RSU, the transmit and receive beams can be accordingly updated and aligned with each other, such that 
TABLE I

INITIAL STATE FOR THE VEHICLE

\begin{tabular}{cccc}
\hline Angle $\theta_{0}$ & Distance $d_{0}$ & Velocity $v_{0}$ & Reflection Coefficient $\beta_{0}$ \\
\hline $9.21^{\circ}$ & $25 \mathrm{~m}$ & $18 \mathrm{~m} / \mathrm{s}$ & $\frac{\sqrt{2}}{2}+\frac{\sqrt{2}}{2} j$ \\
\hline
\end{tabular}

a high-quality communication link can be maintained.

Remark 1-Communication-only benchmark using EKF: The above system model can also be transformed into a pure communication protocol using EKF for beam tracking, where the vehicle performs the prediction and tracking by exploiting the pilots arrived from the RSU, and feeds back the estimated beam as well as the predicted beam of the next time-slot to the RSU. In particular, the state model would remain the same, except that the reflection coefficient $\beta_{n}$ is replaced by the LoS channel coefficient $\alpha_{n}$. The measurement model, however, should be modified as follows. Firstly, the pilot signal will be received by using a receive beamformer at the vehicle, which is formulated by a predicted angle. Secondly, the timedelay $\tau_{n}$ would be a one-way trip delay rather than its roundtrip counterpart. Finally, the velocity can be read from the speedometer rather than from the Doppler frequency.

Remark 2-DFRC vs. communication-only: As can be seen in Fig. 1(c), the advantages of using DFRC are obvious compared to communication-only feedback-based beam training/tracking protocols, which we list as follows.

- First of all, the entire DFRC signal block $s(t)$ is used for both downlink communication and vehicle sensing, where dedicated downlink pilots are no longer needed. This reduces the downlink overheads, while at the same time improving the radar estimates.

- Secondly, the uplink feedback signal is replaced with the echo signal reflected by the vehicle, which reduces the uplink overheads.

- Thirdly, the communication-only scheme requires to quantize the estimated angle before feeding it back to the RSU. In contrast to that, the DFRC scheme can estimate the angle continuously from the echo signal received by the RSU, which improves the estimation accuracy.

- Finally, using the entire DFRC signal block for radar sensing would benefit from the matched-filtering gain $G$, which equals to the energy of $s(t)$. In general, the matched filtering gain of $s(t)$ that spans the whole communication block, is much more significant than that of the feedback based scheme, where only a limited number of pilots are used for beam tracking. As a result, the estimation accuracy can be again improved.

In general, the DFRC transmission scheme provides both SNR gain in beam tracking as well as considerably lower overheads and latency.

\section{NumericAl Results}

In this section, we present a comparative evaluation through indicative numerical results between the SoA beam tracking and DFRC approaches. For more thorough comparisons the reader is referred to [11], [12]. Our benchmark techniques are

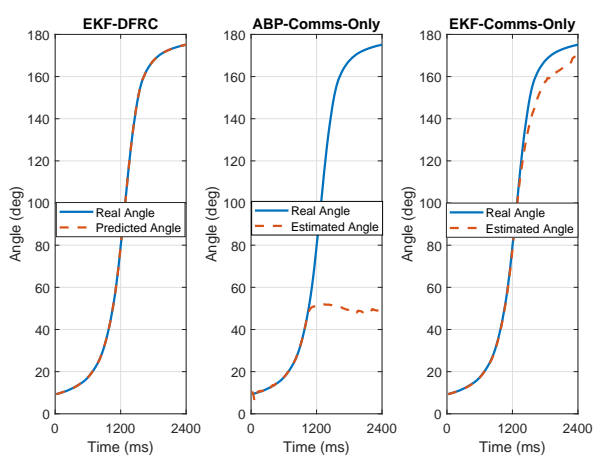

Fig. 3. Angle tracking performance for a single simulation trial.

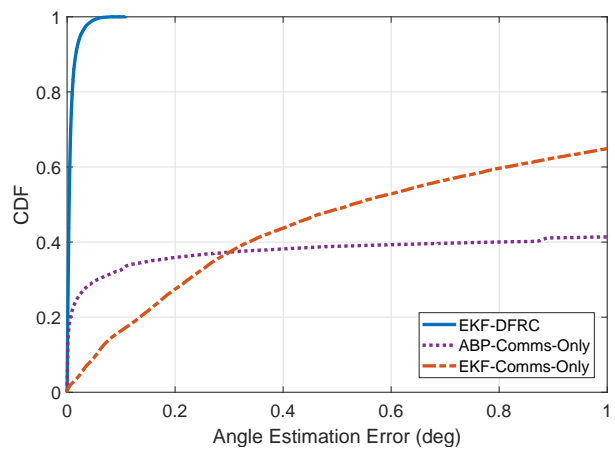

Fig. 4. Overall angle tracking performance.

the ABP method in [10] and the EKF based communicationonly beam tracking in Remark 1 . We consider a V2I scenario where a 64-antenna RSU is serving a 64-antenna vehicle on a straight road, with the initial state $\mathbf{x}_{0}=\left[\theta_{0}, d_{0}, v_{0}, \beta_{0}\right]^{T}$ showing in TABLE. I. The vehicle starts from one side of the RSU, then passes in front of the RSU to the other side. To guarantee a fair comparison between DFRC based and communicationonly schemes, we set the initial channel coefficient $\alpha_{0}=\beta_{0}$. For the DFRC scheme, the matched filtering gain is assumed to be $G=10$. For the ABP scheme, $\Delta \vartheta$ is set as $\frac{\pi}{32}$ without loss of generality. Finally, the transmit SNR is set as $10 \mathrm{~dB}$, and the length of the time-slot is $\Delta T=20 \mathrm{~ms}$.

We first show the angle tracking performance of the DFRC and the communication-only schemes for a single simulation trial. Among all the three techniques, it is notable in Fig. 3 that DFRC is the only technique that correctly tracks the vehicle's angle throughout the vehicle's trajectory. While both the other two techniques are able to keep up with the angular variation at first, they fail to do so at certain points of the road. Fig. 4 shows the overall tracking performance via the cumulative distribution function (CDF) of angle estimation errors. Again, we see that the DFRC significantly outperforms the other two methods, by using which the estimation error can be reduced to below $0.2^{\circ}$.

Finally, we illustrate the average achievable rates for the downlink V2I communication in Fig. 5. We also provide a schematic of the RSU and vehicle's relative position corresponding to the time indices presented in the figure. Intuitively, the communication rate is expected to increase at first and then 


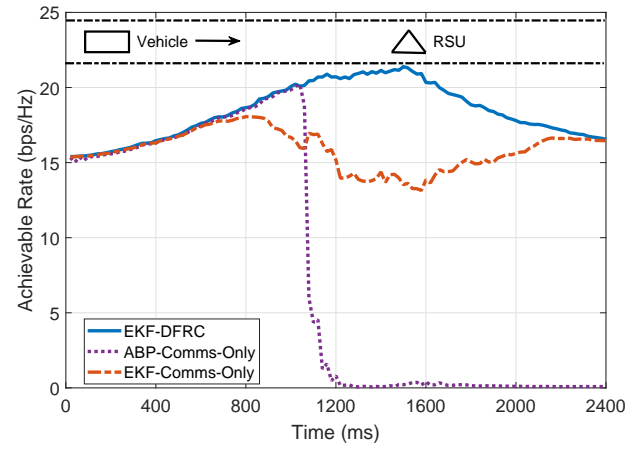

Fig. 5. Average achievable downlink communication rates.

decrease, since the vehicle firstly approaches the RSU and then drives away. Nevertheless, for the high-mobility scenario considered, only the DFRC scheme maintains a relatively stable data rate that matches this trend. For the $\mathrm{ABP}$ technique, it is observed that the rate goes down to zero drastically at $1040 \mathrm{~ms}$. This is because at each transmission slot, the beam pair searches an interval with fixed size, i.e, $2 \Delta \theta=\frac{\pi}{16}$. When the angular variation is large, the correct beam will unlikely fall into this interval, and as a result, the ABP algorithm loses the track. For the EKF based communication-only beam tracking, the rate begins to decrease at $660 \mathrm{~ms}$, and finally catches up with the EKF-DFRC method at 2300ms, which again verifies the superiority of the DFRC scheme.

\section{Conclusion And Open Problems}

\section{A. Conclusion}

In this letter, we have discussed the SoA in DFRC based beam tracking and prediction in the vehicular network. We first introduced the conventional beam training and tracking techniques in the generic cellular network. Following that, we have overviewed a DFRC based predictive beamforming design conceived for the V2I links, and show how the vehicular communication performance benefits from the radar sensing. Our study culminated with the comparative evaluation between the communication-only and DFRC beam tracking approaches, providing evidence of the performance gains of DFRC.

\section{B. Open Problems}

The DFRC based predictive beamforming design is a widely open and exciting research area. While the existing works have proposed some initial beam prediction schemes, a number of future research directions and challenges still remain to be explored, which include but are not limited to:

1) Model nonlinearity: While the predictive beamforming design is realized by the EKF in this letter, it can only deal with models with modest nonlinearity, whose performance may degrade in the case of more complex vehicular signal models. There are numerous other Bayesian filters and algorithms, which can be exploited to address highly nonlinear models. Such techniques include unscented Kalman filtering (UKF), Markov chain Monte Carlo (MCMC) method and particle filtering, by using which the beam tracking performance might be further improved.
2) Reliance on state transition model: In general, the angular variation of the vehicle is predicted based on a specific state transition model, which is typically built upon the kinetic equations of the vehicle as well as the roadway geometry. However, an explicit state model would be rather difficult to obtain in complex traffic environments. It is therefore advantageous to develop advanced prediction methods without specifying a state model. Machine learning solutions may also find applications in this regime.

3) Beam association: The conventional beam tracking algorithms require uplink feedback from the vehicles, where the ID of each vehicle can be embedded into the feedback signal. By leveraging that, the RSU is able to transmit desired information at the correct beams. In the DFRC based predictive beamforming design, however, the beams are extracted from the echo signals from the targets, which contain no ID information. To cope with this issue, it is essential to develop effective beam association algorithm to map each beam to each vehicle, where classic data association algorithms, e.g., joint probabilistic data association (JPDA) filter and multiple hypothesis tracking (MHT), would find applications here.

In light of the above challenges, in Part III of this tutorial, we aim to address the reliance of DFRC beam tracking techniques on state evolution models, by introducing a new prediction technique that circumvents this necessity.

\section{REFERENCES}

[1] F. Liu, C. Masouros, A. Petropulu, H. Griffiths, and L. Hanzo, "Joint radar and communication design: Applications, state-of-the-art, and the road ahead," IEEE Trans. Commun., pp. 1-1, 2020.

[2] H. Wymeersch, G. Seco-Granados, G. Destino, D. Dardari, and F. Tufvesson, "5G mmwave positioning for vehicular networks," IEEE Wireless Commun., vol. 24, no. 6, pp. 80-86, Dec 2017.

[3] J. B. Kenney, "Dedicated short-range communications (DSRC) standards in the united states," Proc. IEEE, vol. 99, no. 7, pp. 1162-1182, Jul 2011.

[4] D. Zhang, A. Li, M. Shirvanimoghaddam, P. Cheng, Y. Li, and B. Vucetic, "Codebook-based training beam sequence design for millimeter-wave tracking systems," IEEE Trans. Wireless Commun. vol. 18, no. 11, pp. 5333-5349, Nov 2019.

[5] V. Va, H. Vikalo, and R. W. Heath, "Beam tracking for mobile millimeter wave communication systems," in 2016 IEEE Global Conference on Signal and Information Processing (GlobalSIP), Dec 2016, pp. 743747.

[6] J. Wang et al., "Beam codebook based beamforming protocol for multiGbps millimeter-wave WPAN systems," IEEE J. Sel. Areas Commun., vol. 27, no. 8, pp. 1390-1399, 2009.

[7] A. Alkhateeb, O. El Ayach, G. Leus, and R. W. Heath, "Channel estimation and hybrid precoding for millimeter wave cellular systems," IEEE J. Sel. Topics Signal Process., vol. 8, no. 5, pp. 831-846, 2014.

[8] Z. Xiao, T. He, P. Xia, and X. Xia, "Hierarchical codebook design for beamforming training in millimeter-wave communication," IEEE Trans. Wireless Commun., vol. 15, no. 5, pp. 3380-3392, 2016.

[9] J. Zhang, Y. Huang, J. Wang, X. You, and C. Masouros, "Intelligent interactive beam training for millimeter wave communications," IEEE Trans. Wireless Commun., in press.

[10] D. Zhu, J. Choi, and R. W. Heath, "Auxiliary beam pair enabled AoD and AoA estimation in closed-loop large-scale millimeter-wave MIMO systems," IEEE Trans. Wireless Commun., vol. 16, no. 7, pp. 4770-4785, 2017.

[11] F. Liu, W. Yuan, C. Masouros, and J. Yuan. (2020) "Radar-assisted predictive beamforming for vehicular links: Communication served by sensing". Submitted to IEEE Trans. Wireless Commun. [Online]. Available: https://arxiv.org/abs/2001.09306

[12] W. Yuan, F. Liu, C. Masouros, J. Yuan, D. W. K. Ng, and N. Gonzalez-Prelcic. (2020) "Bayesian predictive beamforming for vehicular networks: A low overhead joint radar-communication approach". Submitted to IEEE Trans. Wireless Commun. [Online]. Available: https://arxiv.org/abs/2005.07698 